\title{
Minimal-Time Ship Routing
}

\author{
W. E. Bleick and F. D. Faulkner \\ U. S. Naval Postgraduate School, Monterey, Calif.
}

(Manuscript received 22 September 1964)

\begin{abstract}
A recent theory of minimal-time ship routing through time-dependent ocean wave height and direction fields is put to a numerical test by using a series of semidaily analyses furnished by the U. S. Navy Fleet Numerical Weather Facility. The interpolations and integrations required are found to be feasible. A resume of the theory is given.
\end{abstract}

\section{Introduction}

Haltiner, Hamilton and 'Arnason (1962) gave a relaxation method solution to the problem of minimaltime routing of ships through ocean wave height and direction fields dependent on the ship location coordinates only. The theory has been extended by Faulkner (1963) to the case where the wave height and direction depend on time also. The present paper confronts this time-dependent theory with actual wave height and direction analyses from the files of the U.S. Navy Fleet Numerical Weather Facility, and reports on practical problems which had to be solved in a test of the theory.

\section{Polar velocity diagram}

A basic ingredient of the theory is the polar diagram of Fig. 1, giving the ship velocity $v$ as a function of the angle $\theta$ between the ship's heading and the wave direction. A diagram of this kind must be specified for each wave height $H$. The points $L, M$ and $N$ on the diagram correspond to the ship speed $v_{h}$ in head waves, $v_{b}$ in beam waves, and $v_{f}$ in following waves. Empirical curves for these three speeds as functions of wave height $H$ are available in the pioneer work of James (1957). His P2-S2-R2 ship type curves, shown in Fig. 2, have been chosen for use here. They have the appearance of being arcs of hyperbolas, at least approximately. This was confirmed when a least squares analysis showed that all three of $v_{h}, v_{b}$ and $v_{f}$ can be represented closely as functions of $H$ by the hyperbolic arc

$$
c_{1}+c_{2} H-\left[\left(c_{3}+c_{4} H\right)^{2}+\left(c_{1}-c_{0}\right)^{2}-c_{3}^{2}\right]^{\frac{2}{2}}
$$

where $c_{0}$ is the point common to all three speeds when $H=0$. The other four constants are related to the asymptotes $\left(c_{2}+c_{4}\right) H+c_{1}+c_{3}$ and $\left(c_{2}-c_{4}\right) H+c_{1}-c_{3}$. It was decided to construct the polar velocity diagram by fitting an ellipse to the points $L, M$ and $N$. This resulted in semi-principal axes $a=\left(v_{h}+v_{f}\right) / 2, b=a v_{b} /$ $\left(v_{k} v_{f}\right)^{\frac{1}{2}}$, and a distance to the eccentric pole $O$ given by $c=\left(v_{f}-v_{h}\right) / 2$. Note that the pole $O$ is not to be construed as a focus of the ellipse. A further least squares analysis showed that the semiaxes $a$ and $b$ are closely representable also by hyperbolic functions of the form of (1), but not $c$ which must be calculated as $c=\left(v_{f}-v_{h}\right) / 2$.

\section{Coordinate system}

The ocean wave height and direction data of the semidaily Fleet Numerical Weather Facility analyses are presented in a south-polar stereographic projection of the northern hemisphere upon a plane passing through the circle of $60 \mathrm{~N}$ latitude. A rectangular coordinate system is set up in this projecting plane with the $O x$ and $O y$ axes parallel to the projections of the meridians of 10 and $100 \mathrm{E}$ longitude, respectively. A 62 by 62 grid is constructed using these axes with $x=y=31$ defining the projection of the north pole. The mesh distance between grid lines corresponds to a distance of $381 \mathrm{~km}$ at $60 \mathrm{~N}$ latitude where the projection is true. The radius of the equator's projection is 31.205 mesh units. The map scale factor $m$, defined as the ratio of a differential distance in the Oxy plane to the corresponding differential distance on the earth's surface, is

$$
m=\left[973.75+(x-31)^{2}+(y-31)^{2}\right] / 1043.6 .
$$

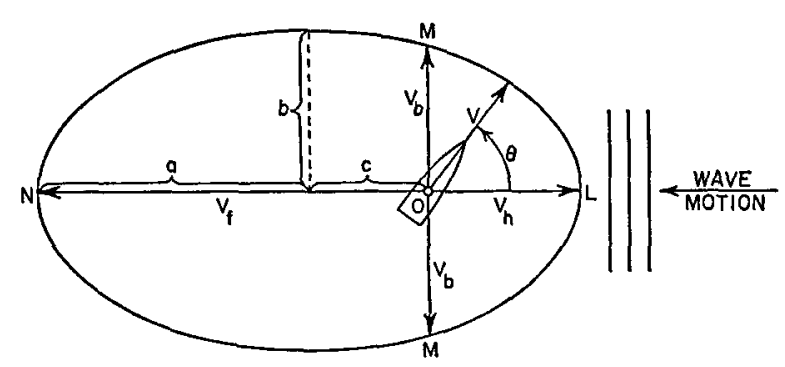

Fig. 1. Polar velocity diagram. 


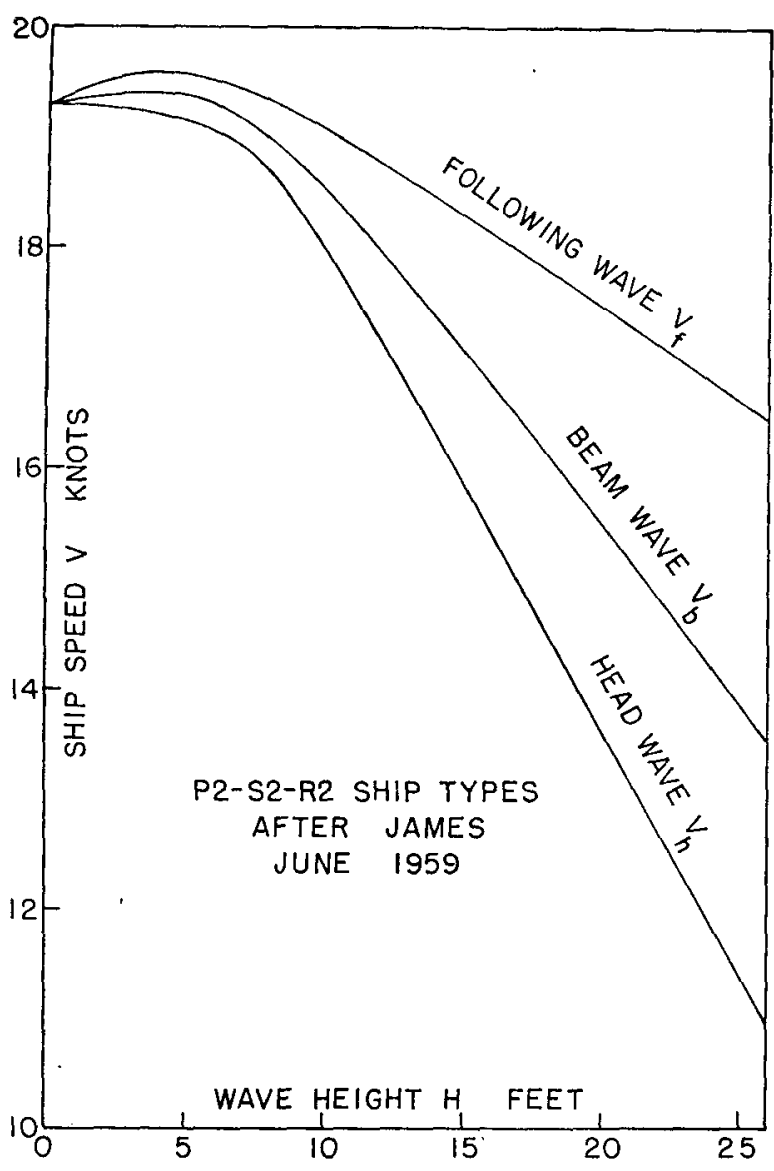

FIG. 2. Ship speed in following, beam and head waves.

Let $x, y$ be the coordinates of a ship's projection in the Oxy plane at time $t$. Then the projected speed of the ship is

$$
V(x, y, t, \theta)=v(H, \theta) m,
$$

where $v(H, \theta)$ is the actual geographical ship speed of the polar velocity diagram of Fig. 1 , and where $H(x, y, t)$ is obtained by interpolation in the Fleet Numerical Weather Facility grid wave height data. Since the stereographic projection is a conformal transformation preserving angles and their senses, the angle $\theta$ is the same in the $O x y$ plane as on the earth's surface.

\section{Resume of the theory}

Fig. 3 shows a ship at the point $(x, y, t)$ in the stereographic projection plane on a route from tixed initial point $A$ at $t=0$ to fixed terminal point $B$ at $t=T$. The elliptical polar velocity diagram for $V=m v$ is plotted at this point by interpolation in the semidaily wave height $H(x, y, l)$ and wave direction $K(x, y, t)$ grid values of the Fleet Numerical Weather Facility. The special type of interpolation required is discussed in the Appendix. The direction of the ship's velocity vector $\mathbf{V}=\mathbf{i} \dot{\mathbf{x}}+\mathbf{j} \dot{y}$ is the control angle $p$, which is to be chosen at each point so as to minimize the transit time $T$ from $A$ to $B$. The equations of motion of the ship's projection in the $O x y$ plane are

$$
\varphi_{1}=\dot{x}-V \cos p=0, \quad \varphi_{2}=\dot{y}-V \sin p=0,
$$

where $V=|\mathbf{V}|=V(x, y, t, p)$. The problem of minimizing $T$ is equivalent to the Lagrange calculus of variations problem of requiring the integral

$$
I=\int_{0}^{T}\left(1+\lambda \varphi_{1}+\mu \varphi_{2}\right) d t
$$

to be stationary, where $\lambda(t)$ and $\mu(t)$ are continua of Lagrangian mutipliers. Let the time at the fixed terminal point $B$ be varied to $T+\Delta T$. The vanishing first variation of $I$ is

$\delta I=\Delta T+[\lambda \delta x+\mu \delta y]_{0}{ }^{T}$

$$
-\int_{0}^{T}\left(\varphi_{3} \delta x+\varphi_{4} \delta y+\varphi_{5} \delta p\right) d t=0 .
$$

The coefficients of $\delta x, \delta y, \delta p$ in $\delta I=0$ give the Euler equations (7), (8) and (9), consisting of the adjoint equations

$$
\begin{aligned}
& \varphi_{3}=\dot{\lambda}+(\lambda \cos p+\mu \sin p) V_{x}=0, \\
& \varphi_{4}=\dot{\mu}+(\lambda \cos p+\mu \sin p) V_{y}=0,
\end{aligned}
$$

and the scalar product control equation

$$
\varphi_{\mathbf{5}}=\boldsymbol{\Lambda} \cdot \mathbf{V}_{p}=\mathbf{0}
$$

where the adjoint vector $\boldsymbol{\Lambda}=\mathbf{i} \lambda+\mathbf{j} \mu$, and where $\mathbf{V}_{p}=\partial \mathbf{V} / \partial p=\mathbf{i}\left(V_{p} \cos p-V \sin p\right)+\mathbf{j}\left(V_{p} \sin p+V \cos p\right)$

is the tangent vector to the polar velocity diagram of Fig. 3. Eq. (9) implies the orthogonality of $\boldsymbol{\Lambda}$ and $\boldsymbol{V}_{p}$ as shown in Fig. 3. Eq. (9) may be written also in the form

$$
p=\arctan (\mu / \lambda)+\arctan \left(V_{p} / V\right) .
$$

The fixed end points $A$ and $B$ imply that

$$
\begin{array}{ll}
d x(0)=\delta x(0)=0, & d y(0)=\delta y(0)=0, \\
d x(T)=(\dot{x} \Delta t+\delta x)_{T}=0, & d y(T)=(\dot{y} \Delta t+\delta y)_{T}=0 .
\end{array}
$$

Use of Eqs. (11) and (12) makes the remaining terms of (6) proportional to $\Delta T$, whose coefficient gives the scalar product transversality condition

$$
(\mathbf{\Lambda} \cdot \mathbf{V})_{T}=1>0
$$

meaningful for sign only because of the homogeneity of (7) and (8). Eq. (13) implies that the angle $(q-p)$ between $\boldsymbol{V}$ and $\boldsymbol{\Lambda}$ is acute, as shown in Fig. 3. A further implication of (10) and (13) is that the quadrant of $p$ is such that

$$
\cos p=\left(\lambda V-\mu V_{p}\right) / \Lambda R, \quad \sin p=\left(\lambda V_{p}+\mu V\right) / \Lambda R,
$$

where $\Lambda=|\boldsymbol{\Lambda}|=\left(\lambda^{2}+\mu^{2}\right)^{\frac{1}{2}}$ and $R=\left|\boldsymbol{V}_{p}\right|=\left(V^{2}+V_{p}^{2}\right)^{\frac{1}{2}}$. 


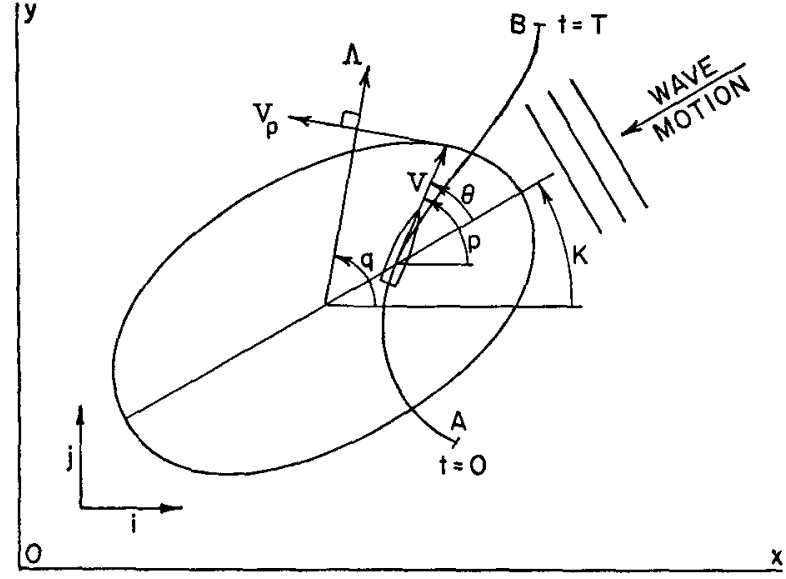

Fig. 3. Ship motion in stereographic $O x y$ plane.

The simultaneous numerical integration of (4), (7), (8) and (14) is carried out together with a NewtonRaphson iteration as follows: Let $\lambda_{1}, \mu_{1}$ and $\lambda_{2}, \mu_{2}$ be two linearly independent solutions of the adjoint Eqs. (7) and (8) corresponding to the columns of the matrix

$$
\mathbf{E}(t)=\left[\begin{array}{ll}
\lambda_{1} & \lambda_{2} \\
\mu_{1} & \mu_{2}
\end{array}\right]
$$

where $\mathbf{E}(0)=\mathbf{I}$ is the identity matrix. The $\lambda, \mu$ of (14) are taken as the linear combinations $\lambda=\lambda_{1} \cos \alpha+\lambda_{2} \sin \alpha$ and $\mu=\mu_{1} \cos \alpha+\mu_{2} \sin \alpha$. The variation $\delta p$ is found by total differentiation of (10) to be

$$
\delta p=R|\mathbf{E}| \delta \alpha / \Lambda^{2}\left(V^{2}+2 V_{p}{ }^{2}-V V_{p p}\right),
$$

where $|\boldsymbol{E}|$ is the determinant of $\mathbf{E}$. Assume that a solution of the ship motion Eqs. (4) has been found, corresponding to (7), (8) and (14) for some value of $T$ and $\alpha$, which falls short of the fixed end point $B$ at $t=T$ by the coordinate differences $\Delta x(T)$ and $\Delta y(T)$. Using this solution and holding $T$ fixed, find the variation of the vanishing matrix integral

$$
\int_{0}^{T}\left[\varphi_{1}, \varphi_{2}\right] \mathbf{E}(t) d t=0
$$

Since the columns of $\mathbf{E}(t)$ satisfy the adjoint Eqs. (7) and ( 8 ), one obtains the $1 \times 2$ matrix equation

$$
\begin{aligned}
{[\delta x, \delta y]_{x} \mathbf{E}(T)=\int_{0}^{T}\left[\left(V_{p} \cos p-V \sin p\right),\right.} \\
\\
\left.\quad\left(V_{p} \sin p+V \cos p\right)\right] \mathbf{E} \delta p d l .
\end{aligned}
$$

Substitution from (14) and (16) into (18) gives

$$
[\delta x, \delta y]_{T}=[-\mu, \lambda]_{T} J \delta \alpha,
$$
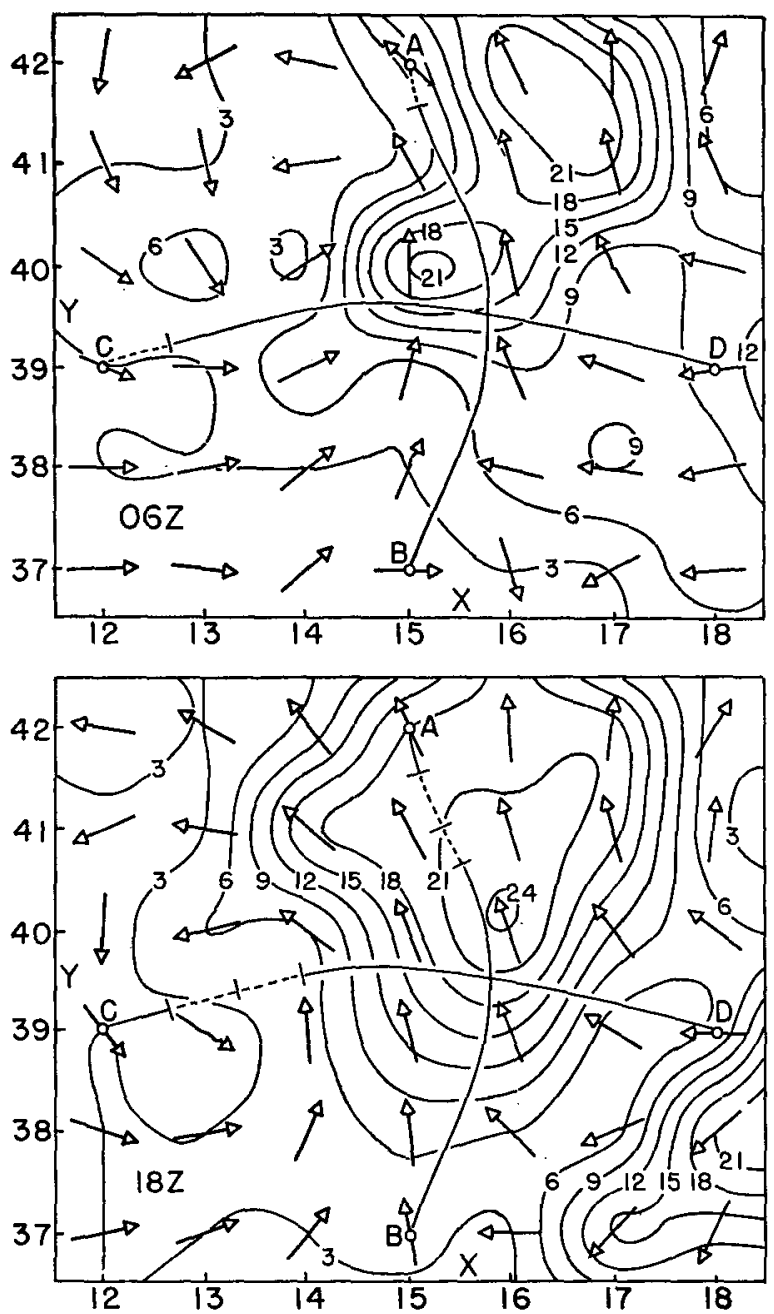

FIg. 4. Sea conditions at $06 Z$ and $18 Z$ on 4 May 1963.

where

$$
J=\frac{1}{|\mathbf{E}(T)|} \int_{0}^{T} R^{3}|\mathbf{E}|^{2} d t / \Lambda^{3}\left(V^{2}+2 V_{p}{ }^{2}-V V_{p p}\right) .
$$

Now vary the terminal time from $T$ to $T+\Delta T$ and substitute

$$
[\delta x, \delta y]_{T}=[\Delta x, \Delta y]_{T}-[\dot{x}, \dot{y}]_{T} \Delta T
$$

into (19) to obtain the Newton-Raphson equations

$$
\begin{aligned}
& \dot{x}(T) \Delta T-J_{\mu}(T) \delta \alpha=\Delta x(T) \\
& \dot{y}(T) \Delta T+J \lambda(T) \delta \alpha=\Delta y(T)
\end{aligned}
$$

for the determination of $\Delta T$ and $\delta \alpha$ on a varied trajectory which attempts to correct the errors $\Delta x(T)$ and $\Delta y(T)$. The iteration to successive varied trajectories is continued until the terminal errors are acceptable. A suitable initial guess for the angle $\alpha$ is the inclination angle of the straight line from $A$ to $B$. 

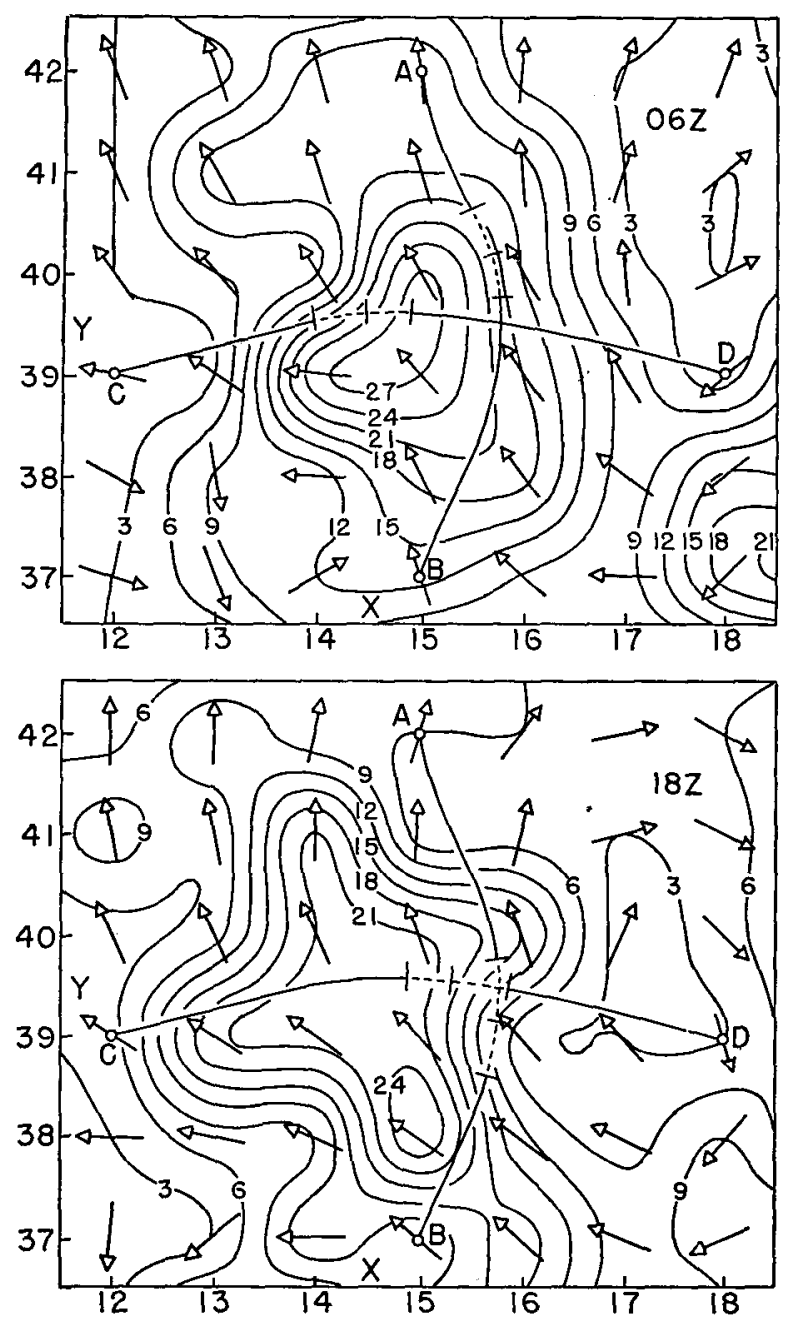

FIG. 5. Sea conditions at 06Z and 18Z on 5 May 1963.

\section{Numerical example}

Ten successive semidaily analyses of wave height and direction, starting at $06 Z$ on 4 May 1963, were furnished by the Fleet Numerical Weather Facility. The maximum speed of the chosen P2-S2-R2 ship type is 19.6 knots. This combination of data precluded a trip of great length. It was decided to select an area of continued extreme wave height for the example. Such an area was found centering at $30 \mathrm{~N}$ latitude and $162 \mathrm{E}$ longitude. Figs. 4, 5 and 6 show two computed minimaltime ship tracks in the area, with contours of wave height in feet and wave direction arrows. The arc traversed by the ship during the 6 hours preceding and/or the 6 hours following the time of each figure is shown as a dashed curve. The minimal-time track $A B$ required 2.488 days with a $3.0 \%$ saving over the geodesic track. The minimal-time track CD required 2.656 days with a $1.3 \%$ saving over the geodesic track. The severity of the sea conditions in the area precludes any more spectacular saving. The highly non-analytic nature of
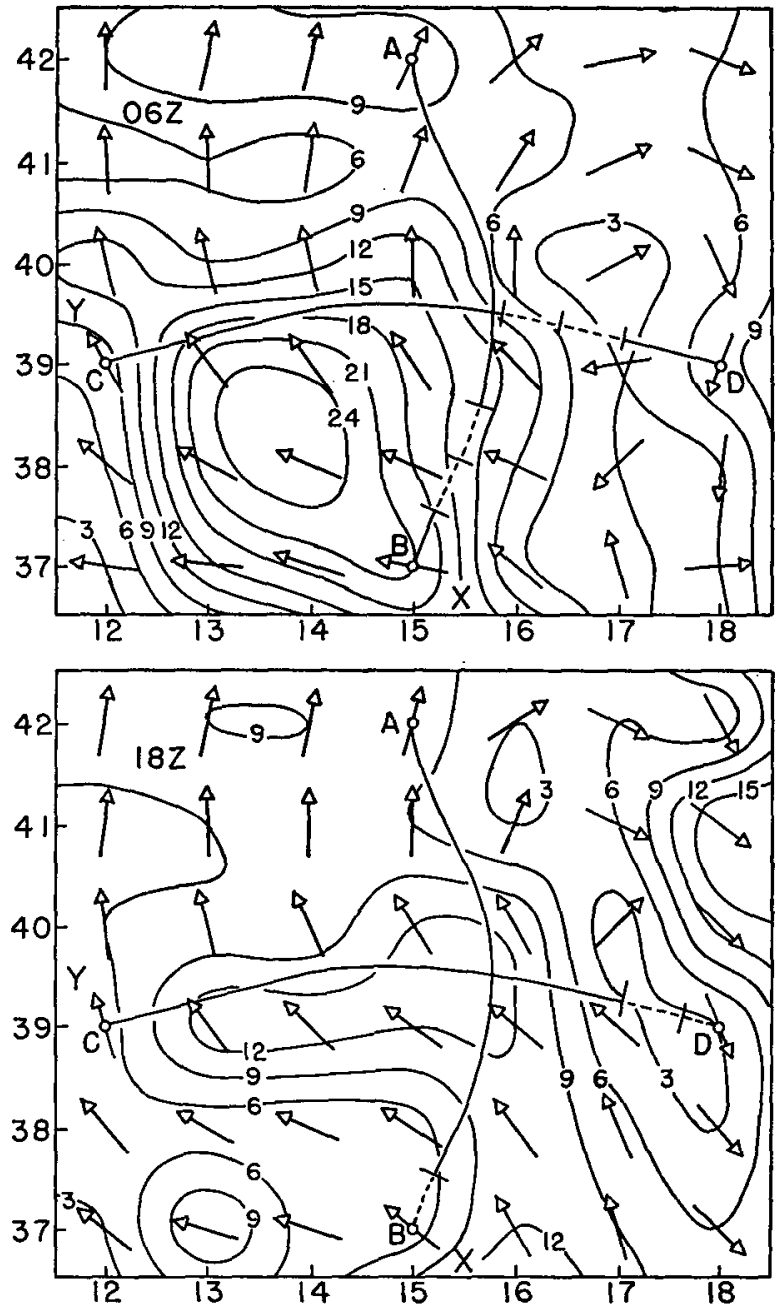

FIG. 6. Sea conditions at $06 Z$ and $18 Z$ on 6 May 1963.

the wave height in the area was found to affect the convergence of the Newton-Raphson iteration of (22). It was found necessary to halve the values of $\Delta T$ and $\delta \alpha$ in order to avoid a divergent oscillation. Resort to this stratagem was found to be unnecessary when the wave height was more nearly analytic.

\section{Concluding remarks}

The numerical integrations involved in the theory of minimal-time ship routing through time-dependent wave fields are found to be feasible. The necessary three-dimensional interpolations in the wave field data, discussed in the Appendix, present no problem. Convergence problems may arise, but can be solved by the described delayed approach to the limit. The authors can supply copies of their Fortran programs for ship routing and for the cubic-interpolation contouring of Figs. 4, 5 and 6.

Wave field forecasts are accurate at present only for a short time range. A method of making the analysis of 
this paper applicable to a long voyage would be to base the minimal-time route on zero wave heights beyond the forecast period, and then compute a new route from the current ship position when new forecast data become available.

Acknowledgments. This work was supported by the Office of Naval Research. Aid from the U. S. Navy Fleet Numerical Weather Facility and from the Computer Facility, U. S. Naval Postgraduate School, is acknowledged.

\section{APPENDIX}

Some pertinent mathematical details are listed here. The geographic wave direction $\nu$, measured clockwise from the North, must be converted to the unit vector $\mathbf{i} \cos K+\mathbf{j} \sin K$ in the stereographic grid system by

$$
\begin{aligned}
& \cos K=-[(x-31) \cos \nu+(y-31) \sin \nu] / r, \\
& \sin K=[(x-31) \sin \nu-(y-31) \cos \nu] / r
\end{aligned}
$$

where $r^{2}=(x-31)^{2}+(y-31)^{2}$. Then the derivative

$$
K_{x}=\cos K(\partial \sin K / \partial x)-\sin K(\partial \cos K / \partial x) .
$$

The derivatives $V_{x}=m v_{x}+v m_{x}$ and $V_{p}=m v_{p}$ are obtained most conveniently by the implicit differentiation of the equation

$$
[v \sin (p-K) / b]^{2}+[(c+v \cos (p-K)) / a]^{2}=1
$$

of the elliptical polar velocity diagram, and noting that $a, b, c$ are functions of $H(x, y, t)$, and that $K$ depends on $x, y, t$. The complexity of the result is reduced by introducing the parameter $\beta$ defined by

$$
\begin{aligned}
& \sin \beta=b \sin (q-K) / s=v \sin (p-K) / b \\
& \cos \beta=a \cos (q-K) / s=[v \cos (p-K)+c] / a,
\end{aligned}
$$

where $s^{2}=a^{2} \cos ^{2}(q-K)+b^{2} \sin ^{2}(q-K)$.
The numerical integration of the adjoint Eqs. (7) and (8) demands an interpolation formula for $B(x, y, l)$, $\cos K(x, y, t)$ and $\sin K(x, y, t)$ which guarantees the continuity of these functions and of their first space and time derivatives where any of $x, y, t$ assume grid values. A 16-point interpolation formula to accomplish this is obtained from the $4 \times 4$ matrix $\mathbf{F}$, whose four rows and columns of function entries correspond to four successive $x$ and $y$ grid values, respectively. The interpolation mesh cell is the central cell of the array, with $x$ and $y$ measured from the cell center, and with the mesh distance considered to be two units. The formula is

$$
F(x, y)=\mathbf{P}(x) \mathbf{F P}^{\prime}(y) / 256,
$$

where the matrix

$$
\begin{array}{r}
P(x)=\left[(1-x)\left(x^{2}-1\right),(x-1)\left(3 x^{2}+2 x-9\right),\right. \\
\left.(x+1)\left(9+2 x-3 x^{2}\right),(x+1)\left(x^{2}-1\right)\right]
\end{array}
$$

and the prime indicates matrix transposition. Interpolation in the time dimension is accomplished by the similar 2-unit-mesh central-difference formula

$$
F(t)=[F(-3), F(-1), F(1), F(3)] \mathbf{P}^{\prime}(t) / 16
$$

which guarantees the continuity of $F(t)$ and $d F / d l$ at each end of the central time interpolation mesh. This formula is consistent with parabolic interpolation at the beginning or end of a time series, where central differences are not available. An interpolated vector $\mathbf{i} \cos K(x, y, t)+\mathbf{j} \sin K(x, y, t)$ should be normalized before use.

\section{REFERENCES}

Haltiner, G. J., H. D. Hamilton and G. 'Arnason, 1962: Minimaltime ship routing. J. Appl. Meteor., 1, 1-7.

Faulkner, F. D., 1963: Numerical methods for determining optimum ship routes. Navigation, 10, 351-367.

James, R. W., 1957 (revised 1959): Application of wave forecasts to marine navigation. U. S. Navy Hydrographic Office, $85 \mathrm{pp}$. 